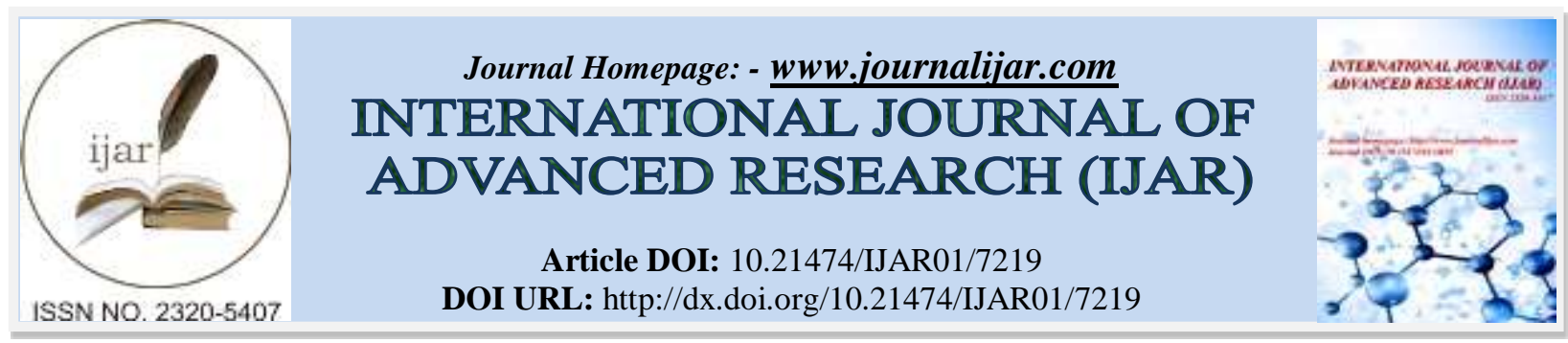

RESEARCH ARTICLE

\title{
LE CAPITAL HUMAIN ENTRE GESTION DU PERSONNEL ET GESTION DES RESSOURCES HUMAINES.
}

Prof. Kamel barbar ${ }^{1}$ and Fatima Roumani ${ }^{2}$.

1. Doyen du Business School Jinan University - Liban.

2. Doctorant en sciences de gestion.

\section{Manuscript Info}

Manuscript History

Received: 06 April 2018

Final Accepted: 08 May 2018

Published: June 2018

\section{Abstract}

Le statut des employés a significativement évolué avec le temps aux yeux de leurs employeurs. En effet, les employés qui étaient considérés essentiellement une source de charges sont perçues de nos jours comme des ressources humaines; un actif à entretenir et développer. En parallèle, l'administration du personnel s'est transformée en gestion du personnel et ensuite s'est métamorphosée en gestion des ressources humaines avec la mise en place de politiques et de pratiques de GRH visant une optimisation de ces ressources et leur rétention. Néanmoins, cette transformation ne s'est pas encore concrétisée dans toutes les entreprises.

La présente étude cherche à examiner à quel point le passage vers une vraie GRH s'est matérialisé au sein des entreprises libanaises. Il s'agit d'une étude de cas d'une entreprise libanaise de l'industrie agroalimentaire dont l'objectif principal est de répondre à la question de recherche suivante : La gestion des affaires des employés dans cette entreprise s'agit-elle d'une authentique GRH ou d'une simple administration du personnel ? Une enquête faite auprès des ressources humaines à l'aide d'un questionnaire a révélé que la société étudiée met en vigueur une gestion du personnel plutôt qu'une gestion de ressources humaines.

Copy Right, IJAR, 2018,. All rights reserved.

\section{Introduction:-}

Une multitude de disciplines s'est penchée sur l'étude du capital humain des organisations. Ces disciplines vont des sciences de gestion des entreprises et de la psychologie passant par d'autres filiales qui ont bien fait couler de l'encre sur ce sujet. Malgré toute l'attention donnée à ce thème, l'intérêt qui lui est donné n'a guère perdu de son élan. Il faut souligner que la résultante principale des premières recherches faites sur le capital humain est la perception des employés comme une source de valeur ajoutée plutôt qu'une source de charges qui pèsent lourd sur l'organisation. Cette résultante a amené les érudits à conceptualiser des politiques et des techniques pour la gestion de cette source de valeur ajoutée. Ces efforts ont abouti au concept de gestion des ressources humaines. L'objectif ultime de ce concept est de mettre à la disposition des organisations une trousse de politiques permettant l'optimisation de la performance des ressources humaines. Ces politiques touchent à toutes les pratiques se rapportant aux employés ; à savoir le recrutement, la rémunération, la formation, etc. 
Le concept de GRH est loin d'être à son stade d'introduction. Néanmoins, sa propagation et son adoption comme substitut à la pratique traditionnelle d'administration du personnel ne se sont pas généralisées dans les organisations et les entreprises. Par suite, les entreprises souffrent toujours de hauts niveaux de turnover, de manque d'optimisation du rendement des employés et d'autres handicaps au niveau des ressources humaines.

Cette recherche se penche sur les pratiques de gestion adoptées en étudiant le cas d'une entreprise libanaise de taille moyenne, MedStar pour l'industrie agroalimentaire, afin de diagnostiquer l'existence ou non de réelles pratiques de GRH. L'objectif final est de répondre aux questions de recherche suivantes : La gestion des affaires des employés dans cette entreprise s'agit-elle d'une authentique GRH ou d'une simple administration du personnel ? Dans le but de répondre notre question de recherche, nous tâcherons à examiner la validité de notre hypothèse de recherche suivante : La société MedStar met en vigueur une administration du personnel plutôt qu'une gestion de ressources humaines.

Pour répondre à ces questions, une étude empirique adoptant une méthodologie quantitative sera menée au sein de cette entreprise. Cette dernière est exécutée par l'administration d'un questionnaire aux employés de la société étudiée.

Ce travail est composé de cinq chapitres. Le premier chapitre est une introduction générale. Le deuxième chapitre offre une revue de la littérature au sujet de l'administration du personnel et de la GRH ainsi que la différenciation entre les deux concepts. Le troisième chapitre dresse les détails de la méthodologie adoptée. Le quatrième chapitre contient les résultats statistiques obtenus. Le cinquième chapitre est une conclusion générale.

\section{Revue de littérature}

\subsection{Evolution de la gestion des employés}

Lorsqu'on passe en revue la définition de la gestion des ressources humaines (GRH) et celle de l'administration ou la gestion du personnel (GP), on réalise que plusieurs différences existent au niveau des perspectives de recherche adoptées. Selon Guest (1990) il existe trois caractéristiques qui paraissent différencier entre la GRH et de la GP.

Premièrement, les approches de GP semblent la considérer comme une fonction de gestion particulièrement destinée aux non-gestionnaires. La GP paraît être appliquée sur les assistants par les gestionnaires et que ces derniers sont accablés par un groupe de codes et de mesures ; limitant ainsi leur liberté dans la gestion de leurs subordonnés (Legge, 2005).

En revanche, la GRH ne consiste pas uniquement à articuler l'importance du développement des membres de l'organisation mais elle consiste à faire développer particulièrement l'équipe de gestion. Par suite, nous pouvons conclure que la GP est une fonction visant essentiellement les non-gestionnaires, alors que la GRH est formellement moins axée sur un groupe spécifique, mais vise principalement le cadre gestionnaire (Guest, 1990).

Deuxièmement, la GP et la GRH mettent en relief la fonction de la gestion de ligne. Dans les formes de GP, le rôle de la ligne correspond largement à l'idée que tous les cadres gèrent le personnel (Storey, 1989). Dans les formes de GRH, les cadres sont supposés travailler ensemble pour gérer le capital humain dans le but d'atteindre les objectifs organisationnels.

Troisièmement, la majorité des formes de GRH soulignent la gestion de la culture d'entreprise comme fonction principale pour le cadre gestionnaire (Köster, 2002). Dans les formes de GP, la culture d'entreprise comme fonction est plutôt externe à la fonction de gestion traditionnelle et est principalement externalisée ou soumise à la charge d'experts en dehors du cadre du service du personnel. C'est le cas contraire dans les modèles de GRH où la gestion de la culture d'entreprise se fait à travers un système intégré de politiques touchant à l'ensemble des pratiques de GRH ; à savoir le recrutement, la rémunération, la gestion des carrières etc. 
Tableau 1:- Hypothèses alternatives sous-jacentes à la GRH

\begin{tabular}{|c|c|c|}
\hline & Conformité & Engagement \\
\hline Contrat psychologique & $\begin{array}{l}\text { Travail quotidien juste pour } \\
\text { une rémunération } \\
\text { quotidienne équitable }\end{array}$ & Engagement réciproque \\
\hline Lieu de contrôle & Externe & Interne \\
\hline Relations de l'employé & $\begin{array}{l}\text { Pluraliste } \\
\text { Collectif } \\
\text { Faible confiance }\end{array}$ & $\begin{array}{l}\text { Unitariste } \\
\text { Individuel } \\
\text { Forte confiance }\end{array}$ \\
\hline Principes organisationnels & $\begin{array}{l}\text { Mécaniste } \\
\text { Rôles formels et définis } \\
\text { Haut vers le bas } \\
\text { Centralisés }\end{array}$ & $\begin{array}{l}\text { Organique } \\
\text { Rôles flexibles } \\
\text { Bas vers le haut } \\
\text { Décentralisés }\end{array}$ \\
\hline Objectifs de politique & $\begin{array}{l}\text { Efficacité administrative } \\
\text { Performance standard } \\
\text { Minimisation des coûts }\end{array}$ & $\begin{array}{l}\text { Main d'œuvre adaptive } \\
\text { Améliorer la performance } \\
\text { Utilisation maximale }\end{array}$ \\
\hline
\end{tabular}

Source : Guest (1990) p.152.

\subsection{Une comparaison entre GP et GRH}

Les différentes comparaisons entre GP et GRH mettent en évidence que la seconde est active et entreprenante ainsi naturellement paraît plus favorable et captivante que la GP qui est souvent associée à la bureaucratie (Beardwell et al., 2004). En parallèle, les preuves témoignant des écarts entre GP et GRH ne sont pas rares. La majorité de ces écarts sont attachées à l'approche flexible (la collaboration des gestionnaires à la culture d'entreprise) et à l'approche ferme (s'intéresser uniquement aux besoins de l'entreprise et les indicateurs de performance financière), l'intégration de la stratégie et l'investissement dans le capital humain dans une perspective future de gestion de carrières, d'avancement et de rétention de ces ressources.

En somme, la GP est axée sur le côté opérationnel. Par suite, elle apprécie les habiletés rattachées aux tâches quotidiennes dans sa décision de recrutement, de formation, de répartition des salaires. En revanche, la GRH est jugée plus dynamique qui voit les employés comme un actif précieux qu'il faut gérer soigneusement afin de le développer comme tout autre actif de l'entreprise. Par suite, on considère que la GRH est au centre de l'activité stratégique de l'organisation puisque c'est elle qui permet la gestion des qualifications des employés dans la direction de l'atteinte des objectifs organisationnels préétablis (Price, 2007).

\section{Méthodologie de recherche}

\subsection{La collecte des données}

L'approche quantitative de la présente étude consiste en une collecte de données par questionnaire. Ce dernier est distribué aux employés de la société MedStar. Il s'intéresse à capter la perception de ces employés et leur évaluation des différentes pratiques de GRH au sein de leur entreprise. La collecte des données vise à savoir dans quelle mesure la société MedStar met en vigueur une vraie GRH ou plutôt une administration de son personnel.

La construction du questionnaire permet aux ressources humaines de la société étudiée de donner leur appréciation des différentes pratiques de GRH au sein de leur organisation. Ces pratiques comprennent ce qui suit :

- Le recrutement et la sélection (RECSEL)

- La formation (FORM)

- La gestion des compétences (GCOMP)

- La rémunération (REM)

- L'équité salariale (EQSAL)

- L'évaluation du rendement (EVREND)

- La gestion de carrière (GCAR) 
En outre, le questionnaire recueille encore des renseignements personnels sur les répondants, notamment démographiques. Ceci est dans le but d'analyser la variation des opinions selon les différentes catégories des répondants.

\subsection{Le traitement des données}

Les données collectées par notre questionnaire sont soumises au logiciel SPSS pour leur traitement et le dépouillement des résultats. La première catégorie de ces derniers est constituée des statistiques descriptives qui sont dépouillées en montrant les moyennes, les écarts types et les coefficients de variation.

La deuxième catégorie des résultats est constituée des statistiques inférentielles qui sont dépouillées en distribuant les données obtenues sur les différentes catégories démographiques de l'échantillon ciblé. Autrement dit, c'est l'examen de l'impact des variables démographiques de l'échantillon sur les réponses des répondants. Le niveau de signification de la variation des réponses selon les catégories démographiques est mesuré à l'aide du test d'ANOVA et de Student T-test.

En parallèle, la validité des échelles choisies dans notre questionnaire a été examinée à l'aide du test Alpha de Cronbach. Ce dernier doit être supérieur à 0,7 pour conclure que la cohérence interne de l'échelle utilisée est forte. En effet, cet indicateur pour les variables RECSEL, FORM, GCOMP, REM, EQSAL, EVREND et GCAR a été de $0.85,0.77,0.73,0.82,0.77,0.70$ et 0.71 respectivement ; reflétant ainsi une forte cohérence interne pour toutes les sept échelles.

4. Résultats et analyse

4.1 Statistiques descriptives

Tableau 4.1 Résultats pour la variable RECSEL

\begin{tabular}{|l|l|l|l|l|}
\hline & Moyenne & Mode & ET & CV \\
\hline Q1 & 2.65 & 3 & 0.98 & $36.79 \%$ \\
\hline Q2 & 2.40 & 3 & 1.04 & $43.46 \%$ \\
\hline Q3 & 2.46 & 3 & 0.89 & $35.98 \%$ \\
\hline RECSEL & 2.50 & 3.00 & 0.85 & $33.89 \%$ \\
\hline
\end{tabular}

Les notes données aux composantes de la variable RECSEL ont reflété tantôt une estimation négative tantôt une estimation neutre de ces composantes. La moyenne des opinions montre que les répondants ont donné une évaluation neutre pour la première composante (Moyenne $\approx 3.00$ ) et une évaluation négative pour les deux autres composantes (Moyenne $\approx 2.00$ ). Les niveaux d'ET et de CV articulent une faible dispersion des évaluations individuelles autour de la moyenne. Au total, les répondants ont donné une évaluation moyenne négative à l'égard de la politique de recrutement et de sélection en tant que pratique de GRH.

Tableau 4.2 Résultats pour la variable FORM

\begin{tabular}{|l|l|l|l|l|}
\hline & Moyenne & Mode & ET & CV \\
\hline Q1 & 2.48 & 3 & 0.92 & $37.10 \%$ \\
\hline Q2 & 2.38 & 3 & 1.03 & $43.11 \%$ \\
\hline Q3 & 2.40 & 3 & 1.00 & $41.54 \%$ \\
\hline FORM & 2.42 & 2.67 & 0.81 & $33.49 \%$ \\
\hline
\end{tabular}

Les notes données aux composantes de la variable FORM ont reflété une estimation négative de ces composantes (Moyenne $\approx 2.00$ ). Les niveaux d'ET et de CV articulent une faible dispersion des évaluations individuelles autour de la moyenne. Au total, les répondants ont donné une évaluation moyenne négative à l'égard de la politique de formation en tant que pratique de GRH.

Tableau 4.3 Résultats pour la variable GCOMP

\begin{tabular}{|l|l|l|l|l|}
\hline & Moyenne & Mode & ET & CV \\
\hline Q1 & 2.46 & 2 & 0.97 & $39.39 \%$ \\
\hline Q2 & 2.32 & 3 & 0.95 & $41.12 \%$ \\
\hline GCOMP & 2.39 & 1.50 & 0.85 & $35.72 \%$ \\
\hline
\end{tabular}


Les notes données aux composantes de la variable GCOMP ont reflété une estimation négative de ces composantes (Moyenne $\approx 2.00$ ). Les niveaux d'ET et de CV articulent une faible dispersion des évaluations individuelles autour de la moyenne. Au total, les répondants ont donné une évaluation moyenne négative à l'égard de la gestion des compétences en tant que pratique de GRH.

Tableau 4.4 Résultats pour la variable REM

\begin{tabular}{|l|l|l|l|l|}
\hline & Moyenne & Mode & ET & CV \\
\hline Q1 & 2.42 & 2 & 0.93 & $38.60 \%$ \\
\hline Q2 & 2.49 & 3 & 0.83 & $33.37 \%$ \\
\hline Q3 & 2.45 & 3 & 0.87 & $35.39 \%$ \\
\hline Q4 & 2.46 & 2 & 0.94 & $38.09 \%$ \\
\hline REM & 2.45 & 2.75 & 0.72 & $29.42 \%$ \\
\hline
\end{tabular}

Les notes données aux composantes de la variable REM ont reflété une estimation négative de ces composantes (Moyenne $\approx 2.00$ ). Les niveaux d'ET et de CV articulent une faible dispersion des évaluations individuelles autour de la moyenne. Au total, les répondants ont donné une évaluation moyenne négative à l'égard de la politique de rémunération en tant que pratique de GRH.

Tableau 4.5 Résultats pour la variable EQSAL

\begin{tabular}{|l|l|l|l|l|}
\hline & Moyenne & Mode & ET & CV \\
\hline Q1 & 2.18 & 1 & 1.04 & $47.89 \%$ \\
\hline Q2 & 2.14 & 1 & 1.06 & $49.49 \%$ \\
\hline Q3 & 2.17 & 2 & 1.01 & $46.50 \%$ \\
\hline Q4 & 2.31 & 2 & 1.07 & $46.49 \%$ \\
\hline Q5 & 2.32 & 2 & 1.08 & $46.42 \%$ \\
\hline Q6 & 2.31 & 2 & 0.97 & $41.86 \%$ \\
\hline EQSAL & 2.24 & 1.67 & 0.70 & $31.40 \%$ \\
\hline
\end{tabular}

Les notes données aux composantes de la variable EQSAL ont reflété une estimation négative de ces composantes (Moyenne $\approx 2.00$ ). Les niveaux d'ET et de CV articulent une faible dispersion des évaluations individuelles autour de la moyenne. Au total, les répondants ont donné une évaluation moyenne négative à l'égard de la politique d'équité salariale en tant que pratique de GRH.

Tableau 4.6 Résultats pour la variable EVREND

\begin{tabular}{|l|l|l|l|l|}
\hline & Moyenne & Mode & ET & CV \\
\hline Q1 & 2.38 & 2 & 0.91 & $38.36 \%$ \\
\hline Q2 & 2.28 & 3 & 1.05 & $46.18 \%$ \\
\hline Q3 & 2.38 & 3 & 1.10 & $46.22 \%$ \\
\hline Q4 & 2.43 & 3 & 1.03 & $42.39 \%$ \\
\hline Q5 & 2.42 & 2 & 0.93 & $38.60 \%$ \\
\hline EVREND & 2.38 & 2.40 & 0.68 & $28.67 \%$ \\
\hline
\end{tabular}

Les notes données aux composantes de la variable EVREND ont reflété une estimation négative de ces composantes (Moyenne $\approx 2.00$ ). Les niveaux d'ET et de CV articulent une faible dispersion des évaluations individuelles autour de la moyenne. Au total, les répondants ont donné une évaluation moyenne négative à l'égard de la politique d'évaluation du rendement en tant que pratique de GRH.

Tableau 4.7 Résultats pour la variable GCAR

\begin{tabular}{|l|l|l|l|l|}
\hline & Moyenne & Mode & ET & CV \\
\hline Q1 & 2.52 & 2 & 1.05 & $41.55 \%$ \\
\hline Q2 & 2.40 & 3 & 1.01 & $42.17 \%$ \\
\hline Q3 & 2.31 & 2 & 1.15 & $49.57 \%$ \\
\hline Q4 & 2.34 & 3 & 1.11 & $47.35 \%$ \\
\hline GCAR & 2.39 & 2.25 & 0.79 & $33.10 \%$ \\
\hline
\end{tabular}


Les notes données aux composantes de la variable GCAR ont reflété tantôt une estimation négative tantôt une estimation neutre de ces composantes. La moyenne des opinions montre que les répondants ont donné une évaluation neutre pour la première composante (Moyenne $\approx 3.00$ ) et une évaluation négative pour les trois autres composantes (Moyenne $\approx 2.00$ ). Les niveaux d'ET et de CV articulent une faible dispersion des évaluations individuelles autour de la moyenne. Au total, les répondants ont donné une évaluation moyenne négative à l'égard de la politique de gestion des carrières en tant que pratique de GRH.

4.1.2 Statistiques inférentielles

Tableau 4.8 Effet de l'âge

\begin{tabular}{|l|l|l|l|l|l|}
\hline & $<22$ & $22-35$ & $36-50$ & $>50$ & Sig \\
\hline RECSEL & 2.32 & 2.47 & 2.50 & 2.94 & 0.262 \\
\hline FORM & 2.17 & 2.45 & 2.55 & 2.73 & 0.257 \\
\hline GCOMP & 2.48 & 2.15 & 2.61 & 2.32 & 0.466 \\
\hline REM & 2.54 & 2.29 & 2.34 & 2.66 & 0.498 \\
\hline EQSAL & 2.09 & 1.99 & 2.51 & 2.59 & $\mathbf{0 . 0 4 0}$ \\
\hline EVREND & 2.10 & 2.39 & 2.53 & 2.75 & 0.051 \\
\hline GCAR & 2.10 & 2.13 & 2.79 & 2.91 & $\mathbf{0 . 0 0 3}$ \\
\hline
\end{tabular}

La comparaison statistique des moyennes montre que celles-ci varient significativement selon l'âge des répondants concernant les variables EQSAL et GCAR. Plus précisément, les individus de plus de 36 ans expriment une évaluation significativement plus positive à l'égard de ces pratiques de la GRH.

Tableau 4.9 Effet du genre

\begin{tabular}{|l|l|l|l|}
\hline & Homme & Femme & Sig \\
\hline RECSEL & 2.60 & 2.35 & 0.244 \\
\hline FORM & 2.58 & 2.16 & 0.040 \\
\hline GCOMP & 2.44 & 2.32 & 0.594 \\
\hline REM & 2.54 & 2.31 & 0.207 \\
\hline EQSAL & 2.23 & 2.25 & 0.941 \\
\hline EVREND & 2.47 & 2.23 & 0.173 \\
\hline GCAR & 2.42 & 2.35 & 0.736 \\
\hline
\end{tabular}

La comparaison statistique des moyennes montre que celles-ci ne varient pas significativement selon le sexe des répondants. Autrement dit, l'évaluation de ces pratiques de GRH n'est pas influencée par le genre des individus.

Tableau 4.10 Effet du niveau d'éducation

\begin{tabular}{|l|l|l|l|l|}
\hline & Secondaire & Licence & Master & Sig \\
\hline RECSEL & 2.61 & 2.20 & 2.92 & $\mathbf{0 . 0 1 4}$ \\
\hline FORM & 2.02 & 2.23 & 3.18 & $\mathbf{0 . 0 0 0}$ \\
\hline GCOMP & 2.19 & 2.17 & 3.00 & $\mathbf{0 . 0 0 2}$ \\
\hline REM & 2.33 & 2.23 & 2.97 & $\mathbf{0 . 0 0 2}$ \\
\hline EQSAL & 2.03 & 2.91 & $\mathbf{0 . 0 0 0}$ \\
\hline EVREND & 1.94 & 2.23 & 2.89 & $\mathbf{0 . 0 0 1}$ \\
\hline GCAR & 2.14 & 2.12 & 3.15 & $\mathbf{0 . 0 0 0}$ \\
\hline
\end{tabular}

La comparaison statistique des moyennes montre que celles-ci varient significativement selon le niveau d'éducation des répondants concernant toutes les variables. Plus précisément, les individus ayant un master expriment une évaluation significativement plus positive à l'égard de ces pratiques de la GRH que les autres individus.

Tableau 4.11 Effet du niveau du poste

\begin{tabular}{|l|l|l|l|l|}
\hline & Niveau d'entrée & Chef & Directeur & Sig \\
\hline RECSEL & 2.35 & 2.37 & 3.94 & $\mathbf{0 . 0 0 0}$ \\
\hline FORM & 2.12 & 2.71 & 3.72 & $\mathbf{0 . 0 0 0}$ \\
\hline GCOMP & 2.08 & 2.74 & 3.58 & $\mathbf{0 . 0 0 0}$ \\
\hline
\end{tabular}




\begin{tabular}{|l|l|l|l|l|}
\hline REM & 2.17 & 2.68 & 3.79 & $\mathbf{0 . 0 0 0}$ \\
\hline EQSAL & 1.94 & 2.44 & 3.72 & $\mathbf{0 . 0 0 0}$ \\
\hline EVREND & 2.11 & 2.46 & 4.03 & $\mathbf{0 . 0 0 0}$ \\
\hline GCAR & 2.02 & 2.69 & 4.17 & $\mathbf{0 . 0 0 0}$ \\
\hline
\end{tabular}

La comparaison statistique des moyennes montre que celles-ci varient significativement selon le niveau du poste des répondants concernant toutes les variables. Plus précisément, les individus ayant un poste de directeur expriment une évaluation significativement plus positive à l'égard de ces pratiques de la GRH que les autres individus.

Tableau 4.12 Effet de l'ancienneté

\begin{tabular}{|l|l|l|l|l|}
\hline & $<3$ & $3-10$ & $>10$ & Sig \\
\hline RECSEL & 2.41 & 2.18 & 3.05 & $\mathbf{0 . 0 2 1}$ \\
\hline FORM & 2.08 & 2.55 & 3.38 & $\mathbf{0 . 0 0 0}$ \\
\hline GCOMP & 2.16 & 2.27 & 3.23 & $\mathbf{0 . 0 0 0}$ \\
\hline REM & 2.22 & 2.57 & 3.10 & $\mathbf{0 . 0 0 0}$ \\
\hline EQSAL & 1.95 & 2.44 & 2.97 & $\mathbf{0 . 0 0 0}$ \\
\hline EVREND & 2.16 & 2.51 & 2.97 & $\mathbf{0 . 0 0 0}$ \\
\hline GCAR & 2.02 & 2.70 & 3.29 & $\mathbf{0 . 0 0 0}$ \\
\hline
\end{tabular}

La comparaison statistique des moyennes montre que celles-ci varient significativement selon l'ancienneté des répondants concernant toutes les variables. Plus précisément, les individus ayant plus de 10 ans d'ancienneté expriment une évaluation significativement plus positive à l'égard de ces pratiques de la GRH que les autres individus.

\subsection{Discussion de l'hypothèse de recherche}

A ce niveau, il est essentiel de récapituler sur les résultats obtenus afin de vérifier la validité de notre hypothèse de recherche et de répondre à notre question de recherche. Pratiquement, les résultats statistiques de l'investigation faite à l'aide du questionnaire administré aux ressources humaines de la société MedStar nous fournissent des preuves empiriques permettant de soutenir et valider notre hypothèse de recherche. En d'autres termes, la société MedStar met en vigueur une administration du personnel plutôt qu'une GRH en bonne et due forme. Nos preuves empiriques sont mises en relief par les évaluations négatives des différentes pratiques de GRH de la part des répondants. Ces résultats confirment que l'implémentation des différentes composantes d'une politique de GRH authentique est loin d'être assurée au sein de la société étudiée.

\section{Conclusions:-}

La revue de littérature a montré que la GRH s'est transformée profusément avec le temps. Elle a évolué d'une administration du personnel primitive vers une stratégie de gestion de ressources humaines à part entière. En conséquence, les stratégies et les pratiques de GRH sont en transformation ininterrompue.

Le but essentiel de cette recherche était d'ausculter le système de GRH à la société MedStar pour certifier qu'il s'agit vraiment d'une GRH ou plutôt d'une simple GP. L'enquête élaborée auprès des employés de la société en question nous a fournit les preuves empiriques nécessaires pour déduire qu'en effet le système adopté est plutôt une GP et non une GRH ; validant ainsi notre hypothèse de recherche.

Les résultats témoignent que le système adopté par la société étudiée échappe aux standards de GRH malgré le niveau de sensibilisation avancé des cadres de cette société qui essaient d'améliorer la fonction de gestion des employés.

\section{Bibliographie:-}

Beardwell, T and Claydon, T (2004). Human Resource management: A contemporary Approach perspective; 5th Ed. Pitman. London.

Guest, D.E. (1990). Personnel Management: The End of Orthodoxy, British Journal of Industrial Relations, Vol. 29, No. 2, pp. 149-176. 
Koster, M (2007). Human resource management versus personnel management. University of Manchester. Available at http://www.solartherm.org/human-resource-management-versus-personnel-management-marcokoster.pdf Legge, K (2005). Human Resource Management - Rhetorics and realities, Macmillan, Basingstoke. Price, A (2007). Human resource management in a business context, 2nd ed., Thomson, London. Storey, J (1989). New perspectives on human resource management, Routlege. London.

Une multitude de disciplines s'est penchée sur l'étude du capital humain des organisations. Ces disciplines vont des sciences de gestion des entreprises et de la psychologie passant par d'autres filiales qui ont bien fait couler de l'encre sur ce sujet. Malgré toute l'attention donnée à ce thème, l'intérêt qui lui est donné n'a guère perdu de son élan. Il faut souligner que la résultante principale des premières recherches faites sur le capital humain est la perception des employés comme une source de valeur ajoutée plutôt qu'une source de charges qui pèsent lourd sur l'organisation.

Cette résultante a amené les érudits à conceptualiser des politiques et des techniques pour la gestion de cette source de valeur ajoutée. Ces efforts ont abouti au concept de gestion des ressources humaines. L'objectif ultime de ce concept est de mettre à la disposition des organisations une trousse de politiques permettant l'optimisation de la performance des ressources humaines. Ces politiques touchent à toutes les pratiques se rapportant aux employés ; à savoir le recrutement, la rémunération, la formation, etc.

Le concept de GRH est loin d'être à son stade d'introduction. Néanmoins, sa propagation et son adoption comme substitut à la pratique traditionnelle d'administration du personnel ne se sont pas généralisées dans les organisations et les entreprises. Par suite, les entreprises souffrent toujours de hauts niveaux de turnover, de manque d'optimisation du rendement des employés et d'autres handicaps au niveau des ressources humaines.

Cette recherche se penche sur les pratiques de gestion adoptées en étudiant le cas d'une entreprise libanaise de taille moyenne, MedStar pour l'industrie agroalimentaire, afin de diagnostiquer l'existence ou non de réelles pratiques de GRH. L'objectif final est de répondre aux questions de recherche suivantes : La gestion des affaires des employés dans cette entreprise s'agit-elle d'une authentique GRH ou d'une simple administration du personnel ? Dans le but de répondre notre question de recherche, nous tâcherons à examiner la validité de notre hypothèse de recherche suivante : La société MedStar met en vigueur une administration du personnel plutôt qu'une gestion de ressources humaines.

Pour répondre à ces questions, une étude empirique adoptant une méthodologie quantitative sera menée au sein de cette entreprise. Cette dernière est exécutée par l'administration d'un questionnaire aux employés de la société étudiée.

Ce travail est composé de cinq chapitres. Le premier chapitre est une introduction générale. Le deuxième chapitre offre une revue de la littérature au sujet de l'administration du personnel et de la GRH ainsi que la différenciation entre les deux concepts. Le troisième chapitre dresse les détails de la méthodologie adoptée. Le quatrième chapitre contient les résultats statistiques obtenus. Le cinquième chapitre est une conclusion générale.

\section{Revue de littérature}

\subsection{Evolution de la gestion des employés}

Lorsqu'on passe en revue la définition de la gestion des ressources humaines (GRH) et celle de l'administration ou la gestion du personnel (GP), on réalise que plusieurs différences existent au niveau des perspectives de recherche adoptées. Selon Guest (1990) il existe trois caractéristiques qui paraissent différencier entre la GRH et de la GP.

Premièrement, les approches de GP semblent la considérer comme une fonction de gestion particulièrement destinée aux non-gestionnaires. La GP paraît être appliquée sur les assistants par les gestionnaires et que ces derniers sont accablés par un groupe de codes et de mesures ; limitant ainsi leur liberté dans la gestion de leurs subordonnés (Legge, 2005).

En revanche, la GRH ne consiste pas uniquement à articuler l'importance du développement des membres de l'organisation mais elle consiste à faire développer particulièrement l'équipe de gestion. Par suite, nous pouvons conclure que la GP est une fonction visant essentiellement les non-gestionnaires, alors que la GRH est formellement moins axée sur un groupe spécifique, mais vise principalement le cadre gestionnaire (Guest, 1990). 
Deuxièmement, la GP et la GRH mettent en relief la fonction de la gestion de ligne. Dans les formes de GP, le rôle de la ligne correspond largement à l'idée que tous les cadres gèrent le personnel (Storey, 1989). Dans les formes de GRH, les cadres sont supposés travailler ensemble pour gérer le capital humain dans le but d'atteindre les objectifs organisationnels.

Troisièmement, la majorité des formes de GRH soulignent la gestion de la culture d'entreprise comme fonction principale pour le cadre gestionnaire (Köster, 2002). Dans les formes de GP, la culture d'entreprise comme fonction est plutôt externe à la fonction de gestion traditionnelle et est principalement externalisée ou soumise à la charge d'experts en dehors du cadre du service du personnel. C'est le cas contraire dans les modèles de GRH où la gestion de la culture d'entreprise se fait à travers un système intégré de politiques touchant à l'ensemble des pratiques de GRH ; à savoir le recrutement, la rémunération, la gestion des carrières etc.

Tableau 1 Hypothèses alternatives sous-jacentes à la GRH

\begin{tabular}{|l|l|l|}
\hline & Conformité & Engagement \\
\hline Contrat psychologique & $\begin{array}{l}\text { Travail quotidien juste pour } \\
\text { une rémunération } \\
\text { quotidienne équitable }\end{array}$ & Engagement réciproque \\
\hline Lieu de contrôle & Externe & Interne \\
\hline Relations de 1'employé & $\begin{array}{l}\text { Pluraliste } \\
\text { Collectif }\end{array}$ & $\begin{array}{l}\text { Unitariste } \\
\text { Individuel }\end{array}$ \\
& Faible confiance & Forte confiance \\
\hline Principes organisationnels & Mécaniste & Organique \\
& Rôles formels et définis & Rôles flexibles \\
& Baut vers le bas & Décentralisés \\
\hline Objectifs de politique & Centralisés & Main d'ouvre adaptive \\
& Efficacité administrative & Améliorer la performance \\
& Performance standard & Utilisation maximale \\
\hline
\end{tabular}

Source : Guest (1990) p.152.

\subsection{Une comparaison entre GP et GRH:-}

Les différentes comparaisons entre GP et GRH mettent en évidence que la seconde est active et entreprenante ainsi naturellement paraît plus favorable et captivante que la GP qui est souvent associée à la bureaucratie (Beardwell et al., 2004). En parallèle, les preuves témoignant des écarts entre GP et GRH ne sont pas rares. La majorité de ces écarts sont attachées à l'approche flexible (la collaboration des gestionnaires à la culture d'entreprise) et à l'approche ferme (s'intéresser uniquement aux besoins de l'entreprise et les indicateurs de performance financière), l'intégration de la stratégie et l'investissement dans le capital humain dans une perspective future de gestion de carrières, d'avancement et de rétention de ces ressources.

En somme, la GP est axée sur le côté opérationnel. Par suite, elle apprécie les habiletés rattachées aux tâches quotidiennes dans sa décision de recrutement, de formation, de répartition des salaires. En revanche, la GRH est jugée plus dynamique qui voit les employés comme un actif précieux qu'il faut gérer soigneusement afin de le développer comme tout autre actif de l'entreprise. Par suite, on considère que la GRH est au centre de l'activité stratégique de l'organisation puisque c'est elle qui permet la gestion des qualifications des employés dans la direction de l'atteinte des objectifs organisationnels préétablis (Price, 2007).

\section{Méthodologie de recherche}

\subsection{La collecte des données}

L'approche quantitative de la présente étude consiste en une collecte de données par questionnaire. Ce dernier est distribué aux employés de la société MedStar. Il s'intéresse à capter la perception de ces employés et leur évaluation des différentes pratiques de GRH au sein de leur entreprise. La collecte des données vise à savoir dans quelle mesure la société MedStar met en vigueur une vraie GRH ou plutôt une administration de son personnel. 
La construction du questionnaire permet aux ressources humaines de la société étudiée de donner leur appréciation des différentes pratiques de GRH au sein de leur organisation. Ces pratiques comprennent ce qui suit :

- Le recrutement et la sélection (RECSEL)

- La formation (FORM)

- La gestion des compétences (GCOMP)

- La rémunération (REM)

- L'équité salariale (EQSAL)

- L'évaluation du rendement (EVREND)

- La gestion de carrière (GCAR)

En outre, le questionnaire recueille encore des renseignements personnels sur les répondants, notamment démographiques. Ceci est dans le but d'analyser la variation des opinions selon les différentes catégories des répondants.

\subsection{Le traitement des données :-}

Les données collectées par notre questionnaire sont soumises au logiciel SPSS pour leur traitement et le dépouillement des résultats. La première catégorie de ces derniers est constituée des statistiques descriptives qui sont dépouillées en montrant les moyennes, les écarts types et les coefficients de variation.

La deuxième catégorie des résultats est constituée des statistiques inférentielles qui sont dépouillées en distribuant les données obtenues sur les différentes catégories démographiques de l'échantillon ciblé. Autrement dit, c'est l'examen de l'impact des variables démographiques de l'échantillon sur les réponses des répondants. Le niveau de signification de la variation des réponses selon les catégories démographiques est mesuré à l'aide du test d'ANOVA et de Student T-test.

En parallèle, la validité des échelles choisies dans notre questionnaire a été examinée à l'aide du test Alpha de Cronbach. Ce dernier doit être supérieur à 0,7 pour conclure que la cohérence interne de l'échelle utilisée est forte. En effet, cet indicateur pour les variables RECSEL, FORM, GCOMP, REM, EQSAL, EVREND et GCAR a été de $0.85,0.77,0.73,0.82,0.77,0.70$ et 0.71 respectivement ; reflétant ainsi une forte cohérence interne pour toutes les sept échelles.

\section{Résultats et analyse}

4.1 Statistiques descriptives

Tableau 4.1 Résultats pour la variable RECSEL

\begin{tabular}{|l|l|l|l|l|}
\hline & Moyenne & Mode & ET & CV \\
\hline Q1 & 2.65 & 3 & 0.98 & $36.79 \%$ \\
\hline Q2 & 2.40 & 3 & 1.04 & $43.46 \%$ \\
\hline Q3 & 2.46 & 3 & 0.89 & $35.98 \%$ \\
\hline RECSEL & 2.50 & 3.00 & 0.85 & $33.89 \%$ \\
\hline
\end{tabular}

Les notes données aux composantes de la variable RECSEL ont reflété tantôt une estimation négative tantôt une estimation neutre de ces composantes. La moyenne des opinions montre que les répondants ont donné une évaluation neutre pour la première composante (Moyenne $\approx 3.00$ ) et une évaluation négative pour les deux autres composantes (Moyenne $\approx 2.00$ ). Les niveaux d'ET et de CV articulent une faible dispersion des évaluations individuelles autour de la moyenne. Au total, les répondants ont donné une évaluation moyenne négative à l'égard de la politique de recrutement et de sélection en tant que pratique de GRH.

Tableau 4.2 Résultats pour la variable FORM

\begin{tabular}{|l|l|l|l|l|}
\hline & Moyenne & Mode & ET & CV \\
\hline Q1 & 2.48 & 3 & 0.92 & $37.10 \%$ \\
\hline Q2 & 2.38 & 3 & 1.03 & $43.11 \%$ \\
\hline Q3 & 2.40 & 3 & 1.00 & $41.54 \%$ \\
\hline FORM & 2.42 & 2.67 & 0.81 & $33.49 \%$ \\
\hline
\end{tabular}

Les notes données aux composantes de la variable FORM ont reflété une estimation négative de ces composantes (Moyenne $\approx 2.00$ ). Les niveaux d'ET et de CV articulent une faible dispersion des évaluations individuelles autour 
de la moyenne. Au total, les répondants ont donné une évaluation moyenne négative à l'égard de la politique de formation en tant que pratique de GRH.

Tableau 4.3 Résultats pour la variable GCOMP

\begin{tabular}{|l|l|l|l|l|}
\hline & Moyenne & Mode & ET & CV \\
\hline Q1 & 2.46 & 2 & 0.97 & $39.39 \%$ \\
\hline Q2 & 2.32 & 3 & 0.95 & $41.12 \%$ \\
\hline GCOMP & 2.39 & 1.50 & 0.85 & $35.72 \%$ \\
\hline
\end{tabular}

Les notes données aux composantes de la variable GCOMP ont reflété une estimation négative de ces composantes (Moyenne $\approx 2.00$ ). Les niveaux d'ET et de CV articulent une faible dispersion des évaluations individuelles autour de la moyenne. Au total, les répondants ont donné une évaluation moyenne négative à l'égard de la gestion des compétences en tant que pratique de GRH.

Tableau 4.4 Résultats pour la variable REM

\begin{tabular}{|l|l|l|l|l|}
\hline & Moyenne & Mode & ET & CV \\
\hline Q1 & 2.42 & 2 & 0.93 & $38.60 \%$ \\
\hline Q2 & 2.49 & 3 & 0.83 & $33.37 \%$ \\
\hline Q3 & 2.45 & 3 & 0.87 & $35.39 \%$ \\
\hline Q4 & 2.46 & 2 & 0.94 & $38.09 \%$ \\
\hline REM & 2.45 & 2.75 & 0.72 & $29.42 \%$ \\
\hline
\end{tabular}

Les notes données aux composantes de la variable REM ont reflété une estimation négative de ces composantes (Moyenne $\approx 2.00$ ). Les niveaux d'ET et de CV articulent une faible dispersion des évaluations individuelles autour de la moyenne. Au total, les répondants ont donné une évaluation moyenne négative à l'égard de la politique de rémunération en tant que pratique de GRH.

Tableau 4.5 Résultats pour la variable EQSAL

\begin{tabular}{|l|l|l|l|l|}
\hline & Moyenne & Mode & ET & CV \\
\hline Q1 & 2.18 & 1 & 1.04 & $47.89 \%$ \\
\hline Q2 & 2.14 & 1 & 1.06 & $49.49 \%$ \\
\hline Q3 & 2.17 & 2 & 1.01 & $46.50 \%$ \\
\hline Q4 & 2.31 & 2 & 1.07 & $46.49 \%$ \\
\hline Q5 & 2.32 & 2 & 1.08 & $46.42 \%$ \\
\hline Q6 & 2.31 & 2 & 0.97 & $41.86 \%$ \\
\hline EQSAL & 2.24 & 1.67 & 0.70 & $31.40 \%$ \\
\hline
\end{tabular}

Les notes données aux composantes de la variable EQSAL ont reflété une estimation négative de ces composantes (Moyenne $\approx 2.00$ ). Les niveaux d'ET et de CV articulent une faible dispersion des évaluations individuelles autour de la moyenne. Au total, les répondants ont donné une évaluation moyenne négative à l'égard de la politique d'équité salariale en tant que pratique de GRH.

Tableau 4.6 Résultats pour la variable EVREND

\begin{tabular}{|l|l|l|l|l|}
\hline & Moyenne & Mode & ET & CV \\
\hline Q1 & 2.38 & 2 & 0.91 & $38.36 \%$ \\
\hline Q2 & 2.28 & 3 & 1.05 & $46.18 \%$ \\
\hline Q3 & 2.38 & 3 & 1.10 & $46.22 \%$ \\
\hline Q4 & 2.43 & 3 & 1.03 & $42.39 \%$ \\
\hline Q5 & 2.42 & 2 & 0.93 & $38.60 \%$ \\
\hline EVREND & 2.38 & 2.40 & 0.68 & $28.67 \%$ \\
\hline
\end{tabular}

Les notes données aux composantes de la variable EVREND ont reflété une estimation négative de ces composantes (Moyenne $\approx 2.00$ ). Les niveaux d'ET et de CV articulent une faible dispersion des évaluations individuelles autour de la moyenne. Au total, les répondants ont donné une évaluation moyenne négative à l'égard de la politique d'évaluation du rendement en tant que pratique de GRH. 
Tableau 4.7 Résultats pour la variable GCAR

\begin{tabular}{|l|l|l|l|l|}
\hline & Moyenne & Mode & ET & CV \\
\hline Q1 & 2.52 & 2 & 1.05 & $41.55 \%$ \\
\hline Q2 & 2.40 & 3 & 1.01 & $42.17 \%$ \\
\hline Q3 & 2.31 & 2 & 1.15 & $49.57 \%$ \\
\hline Q4 & 2.34 & 3 & 1.11 & $47.35 \%$ \\
\hline GCAR & 2.39 & 2.25 & 0.79 & $33.10 \%$ \\
\hline
\end{tabular}

Les notes données aux composantes de la variable GCAR ont reflété tantôt une estimation négative tantôt une estimation neutre de ces composantes. La moyenne des opinions montre que les répondants ont donné une évaluation neutre pour la première composante (Moyenne $\approx 3.00$ ) et une évaluation négative pour les trois autres composantes (Moyenne $\approx 2.00$ ). Les niveaux d'ET et de CV articulent une faible dispersion des évaluations individuelles autour de la moyenne. Au total, les répondants ont donné une évaluation moyenne négative à l'égard de la politique de gestion des carrières en tant que pratique de GRH.

4.1.2 Statistiques inférentielles

Tableau 4.8 Effet de l'âge

\begin{tabular}{|l|l|l|l|l|l|}
\hline & $<22$ & $22-35$ & $36-50$ & $>50$ & Sig \\
\hline RECSEL & 2.32 & 2.47 & 2.50 & 2.94 & 0.262 \\
\hline FORM & 2.17 & 2.45 & 2.55 & 2.73 & 0.257 \\
\hline GCOMP & 2.48 & 2.15 & 2.61 & 2.32 & 0.466 \\
\hline REM & 2.54 & 2.29 & 2.34 & 2.66 & 0.498 \\
\hline EQSAL & 2.09 & 1.99 & 2.51 & 2.59 & $\mathbf{0 . 0 4 0}$ \\
\hline EVREND & 2.10 & 2.39 & 2.53 & 2.75 & 0.051 \\
\hline GCAR & 2.10 & 2.13 & 2.79 & 2.91 & $\mathbf{0 . 0 0 3}$ \\
\hline
\end{tabular}

La comparaison statistique des moyennes montre que celles-ci varient significativement selon l'âge des répondants concernant les variables EQSAL et GCAR. Plus précisément, les individus de plus de 36 ans expriment une évaluation significativement plus positive à l'égard de ces pratiques de la GRH.

Tableau 4.9 Effet du genre

\begin{tabular}{|l|l|l|l|}
\hline & Homme & Femme & Sig \\
\hline RECSEL & 2.60 & 2.35 & 0.244 \\
\hline FORM & 2.58 & 2.16 & 0.040 \\
\hline GCOMP & 2.44 & 2.32 & 0.594 \\
\hline REM & 2.54 & 2.31 & 0.207 \\
\hline EQSAL & 2.23 & 2.25 & 0.941 \\
\hline EVREND & 2.47 & 2.23 & 0.173 \\
\hline GCAR & 2.42 & 2.35 & 0.736 \\
\hline
\end{tabular}

La comparaison statistique des moyennes montre que celles-ci ne varient pas significativement selon le sexe des répondants. Autrement dit, l'évaluation de ces pratiques de GRH n'est pas influencée par le genre des individus.

Tableau 4.10 Effet du niveau d'éducation

\begin{tabular}{|l|l|l|l|l|}
\hline & Secondaire & Licence & Master & Sig \\
\hline RECSEL & 2.61 & 2.20 & 2.92 & $\mathbf{0 . 0 1 4}$ \\
\hline FORM & 2.02 & 2.23 & 3.18 & $\mathbf{0 . 0 0 0}$ \\
\hline GCOMP & 2.19 & 2.17 & 3.00 & $\mathbf{0 . 0 0 2}$ \\
\hline REM & 2.33 & 2.23 & 2.97 & $\mathbf{0 . 0 0 2}$ \\
\hline EQSAL & 1.94 & 2.03 & 2.91 & $\mathbf{0 . 0 0 0}$ \\
\hline EVREND & 2.14 & 2.23 & 2.89 & $\mathbf{0 . 0 0 1}$ \\
\hline GCAR & 2.14 & 2.12 & 3.15 & $\mathbf{0 . 0 0 0}$ \\
\hline
\end{tabular}


La comparaison statistique des moyennes montre que celles-ci varient significativement selon le niveau d'éducation des répondants concernant toutes les variables. Plus précisément, les individus ayant un master expriment une évaluation significativement plus positive à l'égard de ces pratiques de la GRH que les autres individus.

Tableau 4.11 Effet du niveau du poste

\begin{tabular}{|l|l|l|l|l|}
\hline & Niveau d'entrée & Chef & Directeur & Sig \\
\hline RECSEL & 2.35 & 2.37 & 3.94 & $\mathbf{0 . 0 0 0}$ \\
\hline FORM & 2.12 & 2.71 & 3.72 & $\mathbf{0 . 0 0 0}$ \\
\hline GCOMP & 2.08 & 2.74 & 3.58 & $\mathbf{0 . 0 0 0}$ \\
\hline REM & 2.17 & 2.68 & 3.79 & $\mathbf{0 . 0 0 0}$ \\
\hline EQSAL & 1.94 & 2.44 & 3.72 & $\mathbf{0 . 0 0 0}$ \\
\hline EVREND & 2.11 & 2.46 & 4.03 & $\mathbf{0 . 0 0 0}$ \\
\hline GCAR & 2.02 & 2.69 & 4.17 & $\mathbf{0 . 0 0 0}$ \\
\hline
\end{tabular}

La comparaison statistique des moyennes montre que celles-ci varient significativement selon le niveau du poste des répondants concernant toutes les variables. Plus précisément, les individus ayant un poste de directeur expriment une évaluation significativement plus positive à l'égard de ces pratiques de la GRH que les autres individus.

Tableau 4.12 Effet de l'ancienneté

\begin{tabular}{|l|l|l|l|l|}
\hline & $<3$ & $3-10$ & $>10$ & Sig \\
\hline RECSEL & 2.41 & 2.18 & 3.05 & $\mathbf{0 . 0 2 1}$ \\
\hline FORM & 2.08 & 2.55 & 3.38 & $\mathbf{0 . 0 0 0}$ \\
\hline GCOMP & 2.16 & 2.27 & 3.23 & $\mathbf{0 . 0 0 0}$ \\
\hline REM & 2.22 & 2.57 & 3.10 & $\mathbf{0 . 0 0 0}$ \\
\hline EQSAL & 1.95 & 2.44 & 2.97 & $\mathbf{0 . 0 0 0}$ \\
\hline EVREND & 2.16 & 2.51 & 2.97 & $\mathbf{0 . 0 0 0}$ \\
\hline GCAR & 2.02 & 2.70 & 3.29 & $\mathbf{0 . 0 0 0}$ \\
\hline
\end{tabular}

La comparaison statistique des moyennes montre que celles-ci varient significativement selon l'ancienneté des répondants concernant toutes les variables. Plus précisément, les individus ayant plus de 10 ans d'ancienneté expriment une évaluation significativement plus positive à l'égard de ces pratiques de la GRH que les autres individus.

\subsection{Discussion de l'hypothèse de recherche}

A ce niveau, il est essentiel de récapituler sur les résultats obtenus afin de vérifier la validité de notre hypothèse de recherche et de répondre à notre question de recherche. Pratiquement, les résultats statistiques de l'investigation faite à l'aide du questionnaire administré aux ressources humaines de la société MedStar nous fournissent des preuves empiriques permettant de soutenir et valider notre hypothèse de recherche. En d'autres termes, la société MedStar met en vigueur une administration du personnel plutôt qu'une GRH en bonne et due forme. Nos preuves empiriques sont mises en relief par les évaluations négatives des différentes pratiques de GRH de la part des répondants. Ces résultats confirment que l'implémentation des différentes composantes d'une politique de GRH authentique est loin d'être assurée au sein de la société étudiée.

\section{Conclusions:-}

La revue de littérature a montré que la GRH s'est transformée profusément avec le temps. Elle a évolué d'une administration du personnel primitive vers une stratégie de gestion de ressources humaines à part entière. En conséquence, les stratégies et les pratiques de GRH sont en transformation ininterrompue.

Le but essentiel de cette recherche était d'ausculter le système de GRH à la société MedStar pour certifier qu'il s'agit vraiment d'une GRH ou plutôt d'une simple GP. L'enquête élaborée auprès des employés de la société en question nous a fournit les preuves empiriques nécessaires pour déduire qu'en effet le système adopté est plutôt une GP et non une GRH ; validant ainsi notre hypothèse de recherche. 
Les résultats témoignent que le système adopté par la société étudiée échappe aux standards de GRH malgré le niveau de sensibilisation avancé des cadres de cette société qui essaient d'améliorer la fonction de gestion des employés.

\section{Bibliographie:-}

1. Beardwell, T and Claydon, T (2004). Human Resource management: A contemporary Approach perspective; 5th Ed. Pitman. London.

2. Guest, D.E. (1990). Personnel Management: The End of Orthodoxy, British Journal of Industrial Relations, Vol. 29, No. 2, pp. 149-176.

3. Koster, M (2007). Human resource management versus personnel management. University of Manchester. Available at http://www.solartherm.org/human-resource-management-versus-personnel-management-marcokoster.pdf

4. Legge, K (2005). Human Resource Management - Rhetorics and realities, Macmillan, Basingstoke.

5. Price, A (2007). Human resource management in a business context, 2nd ed., Thomson, London.

6. Storey, J (1989). New perspectives on human resource management, Routlege. London. 